\title{
Anxiety during COVID-19 among Saudi Arabian Population: A Systematic Review
}

\author{
Mohammed M Al Moaleem ${ }^{\circledR}$
}

\begin{abstract}
Aim: To evaluate the level of anxiety during COVID-19 pandemic status and to investigate the association between gender, level of education, and age-group with the level of anxiety.

Materials and methods: Studies measured anxiety during COVID-19 pandemic in Saudi Arabia via various social media using different indexes. Name of researcher and year of publication, gender, scale used, degree of anxiety, age-groups, and educational level were assessed.

Results: Fifteen studies were involved in this review, and they showed that mild anxiety during COVID-19 was the highest among Saudi patients (54\%), followed by moderate (32\%) and severe (13\%) anxiety. Additionally, the percentages of females, young age-groups, and educated participants were higher than those of their counterparts. Healthcare workers (HCWs) recorded a higher percentage than others during the pandemic.

Conclusion: Overall, during the COVID-19 pandemic, the percentage of moderate and severe levels of anxiety were high. Females and lower age-groups recorded high levels of anxiety than others. Different healthcare and educational programs must be started to minimize the level of anxiety and number of cases.

Clinical significance: To overcome this problem, HCWs should undertake educational sessions with training to help and minimize their patients' level of anxiety. Ministry of Health and Governmental hospitals should build a treatment plan and programs that aids in treatment and recovery of the patients. Also, the importance of anxiety control measures through and for the HCW population should be focused upon. Keywords: Anxiety, Anxiety index, COVID-19, Gender, Saudi Arabia.

World Journal of Dentistry (2022): 10.5005/jp-journals-10015-1889
\end{abstract}

\section{INTRODUCTION}

Anxiety is a general state in which a person experiences a level of apprehension and suspects something bad will happen during a heath visit. ${ }^{1}$ Anxiety, depression, and stress levels are measured as significant indicators of psychological health, and the inability to detect and address these mental disorders negatively affects subjects. ${ }^{2,3}$ COVID-19, which stands for coronavirus disease 2019, is an acute respiratory illnesses that first occurred in Wuhan, Hubei Province, China, in December 2019. ${ }^{4}$ COVID-19 has affected everybody physically and emotionally, which may have led to vulnerability, sleep deprivation, outrage, fear of contamination, increased consumption of liquor or cigarettes, social separation, increase in post-pressure harmful effects, nervousness issues, burdensome confusion, somatization, and loss of wellbeing. ${ }^{5}$

Globally, at 5:19 pm CEST, August 2, 2021, there have been $198,234,951$ confirmed cases of COVID-19, including 4,227,359 deaths, as reported by WHO. ${ }^{6}$ At the end of July 2021, a total of $3,839,816,037$ vaccine doses have been administered worldwide. ${ }^{7}$ In early March 2020, the first case of COVID-19 was confirmed in Saudi Arabia (SA), and since then, it has caused 8259 deaths among the 5,271,877 patients who were infected with this disease until July 2, 2021 (Channel 2021 and World Status). ${ }^{8}$ At that time, $27,382,111$ of vaccination doses have been administered in SA. In comparison to the Gulf and other neighboring countries, SA was ranked on the top after Iran in terms of the total number of COVID-19 confirmed cases and the lowest four with regard to cases of death $(0.60 \%) ., 6,10$

The scales and indexes that have been used to assess the anxiety during COVID-19 were Generalized Anxiety Disorder-7 (GAD-7), ${ }^{11-13,18-20}$ Patient Health Questionnaire (PHQ-9), ${ }^{11,12,18}$ Fear
Department of Prosthetic Dental Science, College of Dentistry, Jazan University, Jazan, Saudi Arabia; College of Dentistry, Ibn al-Nafis University, Sanaa, Yemen

Corresponding Author: Mohammed M Al Moaleem, Department of Prosthetic Dental Science, College of Dentistry, Jazan University, Jazan, Saudi Arabia; College of Dentistry, Ibn al-Nafis University, Sanaa, Yemen, e-mail: drmoaleem2014@gmail.com

How to cite this article: Al Moaleem MM. Anxiety during COVID-19 among Saudi Arabian Population: A Systematic Review. World J Dent 2022;13(1):87-94.

Source of support: Nil

Conflict of interest: None

of COVID-19 Scale (FCV-19S), ${ }^{14}$ Anxiety and Fear Index, ${ }^{15,16}$ WHO Index, ${ }^{17}$ Zung Self-rating Anxiety Scale (Zung S-RAS), ${ }^{21}$ and Q-Platform developed by SharikHealth. ${ }^{22}$ Other indexes such as Depression, Anxiety, and Stress Scale (DASS-21) ${ }^{23,24}$ and Hospital Anxiety and Depression Scale (HADS) ${ }^{25}$ were also used.

The level of anxiety during teeth treatment was considered as moderate to high among Saudi people. ${ }^{26-29}$ While, SA populations were at higher risk of developing mental illness and serious concern during the COVID-19 pandemic. ${ }^{11-15}$ Latest studies that have been published, all of them conclude and recommend the following: considerable attention is required from policymakers, mental healthcare providers, authorities, and government agencies regarding early detection, continuous health support, and treatment of the disease to reduce the burden of the pandemicrelated mental illness. Effective decisions should be made to address students' anxiety as a serious problem, ${ }^{10,11}$ and social systems must

(c) The Author(s). 2022 Open Access This article is distributed under the terms of the Creative Commons Attribution 4.0 International License (https://creativecommons. org/licenses/by-nc/4.0/), which permits unrestricted use, distribution, and non-commercial reproduction in any medium, provided you give appropriate credit to the original author(s) and the source, provide a link to the Creative Commons license, and indicate if changes were made. The Creative Commons Public Domain Dedication waiver (http://creativecommons.org/publicdomain/zero/1.0/) applies to the data made available in this article, unless otherwise stated. 
be in place to minimize the impact of the pandemic. ${ }^{12,13,14}$ Several conclusions focus on the solutions to minimize anxiety during COVID-19 pandemic. ${ }^{18,19,22,23}$

Many places in SA provided health treatments, such as specialized health centers, outpatient clinics, and primary health institutes. Issues related to COVID-19 were recorded in several health provider institutes and different clinical offices. Anxiety can be recorded among university students and health workers/ practitioners (HWPs). Hence, the objectives of these studies are to evaluate the level of anxiety during COVID-19 pandemic and to investigate the associated factors like gender, level of education, monthly income, and age-group with the level of anxiety.

\section{Methods}

This review was designed and planned according to the steps in Preferred Reporting Items for Systematic Review (PRISMA) Moher et al. ${ }^{30}$ We also followed the phases stated in a systematic review published by Herrero-Hernández et al. ${ }^{31}$

\section{Research Design}

The search plan was conducted using the state, context, and population framework and on the basis of the following question: "What is the level of anxiety (stress) among the Saudi population during COVID-19 pandemic?" To answer this question, the condition was the level of anxiety associated with stress in the presence of COVID-19. Only studies that used questionnaires describing the anxiety, depression, and stress indexes during COVID-19 were included. The content mainly included online surveys and some interviews in all the cross-sectional studies carried out using the aforementioned indexes among Saudi participants. The population consisted of participants who had been subjected to stress/anxiety/ depression regardless of its diagnostic purposes.

\section{Search Process for the Studies}

All peer-reviewed original research articles were selected. A bibliographic search of the PubMed, Science Direct, Scopus Index, Wiley Library, Google-Search, and Electronic Databases in Web of Science was done to find the closely related studies from the beginning of COVID-19 attack (March 2020) until August 2, 2021. The purpose was to identify cross-sectional studies where anxiety was assessed and measured using any of the aforementioned indexes. The last update was made on August 29, 2021. The search terms were "anxiety," "stress," "depression," "scale," "indexes," and "Saudi Population". The keywords were used individually or in combination, using "AND," "OR," and "NOT" to independently search for the term "anxiety," include all terms, or exclude "Saudi participants" because of the possibility of finding studies that evaluated anxiety worldwide.

\section{Inclusion and Exclusion Criteria}

Covered services that evaluated anxiety by using any scales or indexes with several points evaluating stress, depression, and anxiety among Saudi patients and that were published in English were included. Furthermore, the association between anxiety, depression, and stress during the COVID-19 pandemic and patients suffering from the disease or their relative, friend, family, or co-worker was evaluated. Exclusion criteria were published studies that did not assess anxiety among Saudi patients, longitudinal study, case control, systemic reviews, case reports, and replica studies. Published studies that did not specify the number of subjects and participants involved in the study, or whose samples had been partly evaluated in other involved studies were excluded.

\section{Data Extraction and Analysis}

The investigator M.A. started reading of all collected related studies abstracts, and those that were not relevant to the search criteria were removed. Next, the aforementioned investigator conducted an independent assessment, according to the inclusion/exclusion criteria, until reaching a harmony on the studies to be involved in the review. Lastly, a total of 89 studies were included, and the full texts of the particular studies were gathered for review. The extracted data from every study was extracted and tabulated as planned by investigator in the following groupings: name of researcher; publication year; city or province name; sample size; relation to COVID-19; type and gender of the applicants; index or scale used; anxiety level, depression, and stress; and significant differences between genders, highest age-groups, marital status, level of education, and monthly income.

\section{Quality of the Reports of the Selected Studies}

We used the STROBE recommendation checklist adapted by researchers for cross-sectional studies on stress/anxiety/and or depression throughout COVID-19 and used any mentioned type of index or scale as a proxy indicator of quality. The importance of the checklist is to evaluate limitations and risk of bias in the published papers, which can lead to an inaccurate interpretation of the results. Although, it can increase the validity and strength of the conclusions and the reproducibility of the process, and it is a pointer that improves the overall quality of the included and related studies. Each study with their indexes used was reviewed by investigator M.A. who carried out a complete count of all the studies involved in this review. Table 1 shows the studies carried out during COVID-19 to evaluate the anxiety levels among SA's population.

\section{Statistical Analysis}

All data were tabulated properly and means were calculated. An evaluation of variables such as relation of anxiety to COVID-19; gender of the participants; type of index or scale used; level of anxiety, depression, and stress; and significant differences between gender, highest age-groups, and marital status was performed among the five regions.

\section{Results}

\section{Studies' Features and Selection}

A total of 89 studies were selected and evaluated. Of these, 54 were removed because of them being duplicates or not related to the study. Of the remaining 35 studies, 20 were removed due to lack of relevance, not related to Saudi populations, or being case studies or systematic reviews, ending with only 15 studies (Fig. 1, Flowchart). The parameters included are in relation to COVID-19; occupation/ job/type and gender of the participants; type of index or scale used; level of anxiety, depression, and stress; and significant differences between gender; highest age-groups, marital status, and level of education (Table 1).

\section{Value of the Reports of the Involved Studies}

All of the 15 studies and surveys conducted during COVID-19 were online using different social medias, and between $100 \%$ and $60 \%$ 


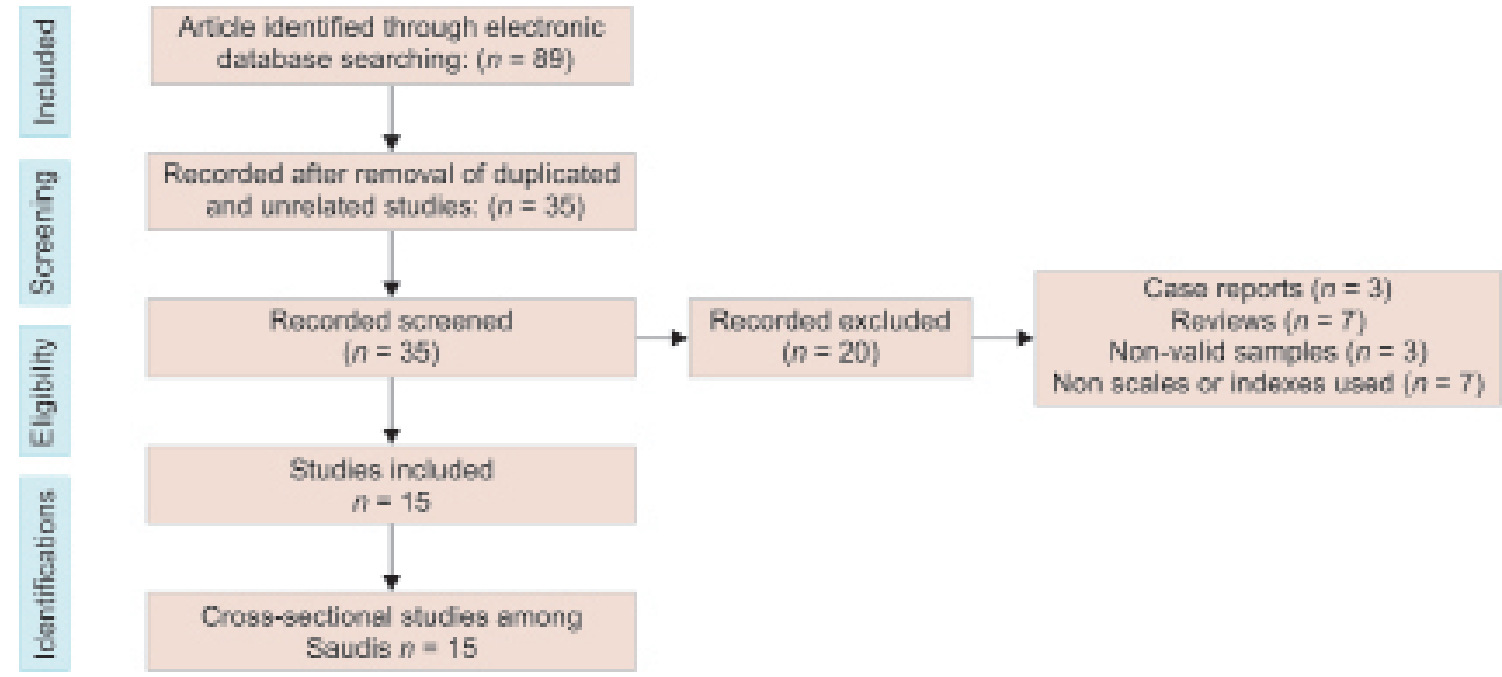

Fig. 1: Study selection process flowchart. PRISMA (Preferred Reporting Items for Scoping/Systematic Review) ${ }^{26,27}$

of the items were reported in all published studies. Among the lowest scores were the items of sample size calculation or pilot studies, reliability, and generalizability, which were presented in $60 \%$ only. Other items such as potential sources of bias, outcome interpretation, and strengths and limitations were recorded in $67 \%$. Furthermore, an interesting note is that the studies during the COVID 19 filled most of the items and covered most of the cities in SA (Table 2).

\section{Synthesis of Studies Included}

Group of studies published during the COVID-19 pandemic assessed the anxiety, depression, and stress of Saudi patients. These studies were cross-sectional and online using the different social medias. Some of them were conducted in all regions of SA, ${ }^{11-14,16,22,23,25}$ in Qassim, ${ }^{15}$ in Jeddah, ${ }^{17}$ in Riyadh, ${ }^{18,19,21}$ in Makkah, ${ }^{20}$ and in Hail. ${ }^{24}$ Most of these studies used stress, depression, and anxiety indexes and scales such as GAD-7, PHQ-9, and DASS-21 (Table 1). The mean percentage of the mild anxiety level was 54\% (ranging between $9 \%$ and $83.6 \%$ ), whereas that of moderate anxiety level was $32 \%$ (from $7 \%$ to $77 \%$ ), but for the high or severe level of anxiety it was $13 \%$.

The percentage of male participants was varied and was found as low as $5.7 \%{ }^{17}$ or reached over $85 \%$ in some studies..$^{11,14}$ However, the percentages of female participants were high and accounted as $94.3 \%{ }^{17}$ and also as low as $15 \% .^{11}$ In general, the average percentage of male to females were either over $70 \%$ or around $10 \%$. There was a significant difference between gender, which was represented in seven studies, males were significant in two studies, and two studies noted non-significant difference. ${ }^{16,17}$ Four studies showed equal rate between genders. ${ }^{11,14,20,25}$ Anxiety was high among the low age-groups as $19-31$ and $31-40$ in most of the studies. Eight studies recorded significant differences between age-groups, whereas five studies ${ }^{11,12,15,21,25}$ recorded nonsignificant differences. Regarding association between anxiety during COVID-19 and educational level, 11 studies showed a significant difference between the two parameters, whereas three studies noted non-significant difference. ${ }^{12,15,20}$ Finally, anxiety was higher in all conducted studies ${ }^{12,16,22,23}$ among different health care worker (HCW) groups in comparison to other groups during pandemic.

\section{Discussion}

Anxiety is one of the most common causes of fear that ultimately leads the general patients to escape and cancel their appointments in different specialized centers, primary health care, and hospitals. ${ }^{26-29}$ COVID-19 is a life-threatening infectious agent that has spread across the world and has become a global concern. This disease was first identified in Wuhan on December 12, 2019. ${ }^{32}$

Anxiety levels among Saudi population is high. ${ }^{26-29}$ Wide range of Saudi residents are at higher risk of developing mental illness during the current COVID-19 pandemic. ${ }^{11-15}$ Policymakers and mental healthcare providers are advised to provide continuous monitoring of the psychological consequences during this pandemic and provide the required health support. ${ }^{11,14}$ Overall, the anxiety level was near to the margin of $50 \%$ of the assessed participants (32\% had moderate anxiety and $13 \%$ had severe anxiety). This percentage is considered very high, and the reason for this high percentage is related to condition of the cities as well as hospitals and different media news. The importance of having a HCW team to provide accurate data from principal foundations needs to be highlighted. The results agree with earlier systematic review that explored the impact of COVID-19 on mental health and anxiety, which ranged from $6.3 \%$ to $50.9 \%$ in countries worldwide such as Turkey, Iran, China, USA, Spain, Italy, Denmark, and Nepal. ${ }^{33}$

Anxiety signs during COVID-19 among the SA participants were more likely to arise in females than males as shown in Table 1. ${ }^{12,18,19,21-24}$ Such outcomes reached consensus and in parallel with the finding of a survey conducted by Al-Khathami et al., ${ }^{34}$ who reported that the prevalence of minor mental illnesses including anxiety is significantly more in females $(22.2 \%)$ than in males (13.7\%), with significant differences. Significant differences related with higher level of anxiety among females were also recorded in many Saudi studies. ${ }^{13,18,24}$ This was confirmed in a previous metaanalysis that explored the prevalence of anxiety across 30 countries all over the world between 1994 and 2014. In this study, females showed higher anxiety rates (14.4\%) compared with males (11.5\%). ${ }^{35}$ Albagmi et al. and Shaikh et al. stated that anxiety during COVID-19 were higher among males, whereas females reported a low level of anxiety. ${ }^{13,15}$ But, when comparing worldwide studies, females usually recorded higher percentage of anxiety in comparison 


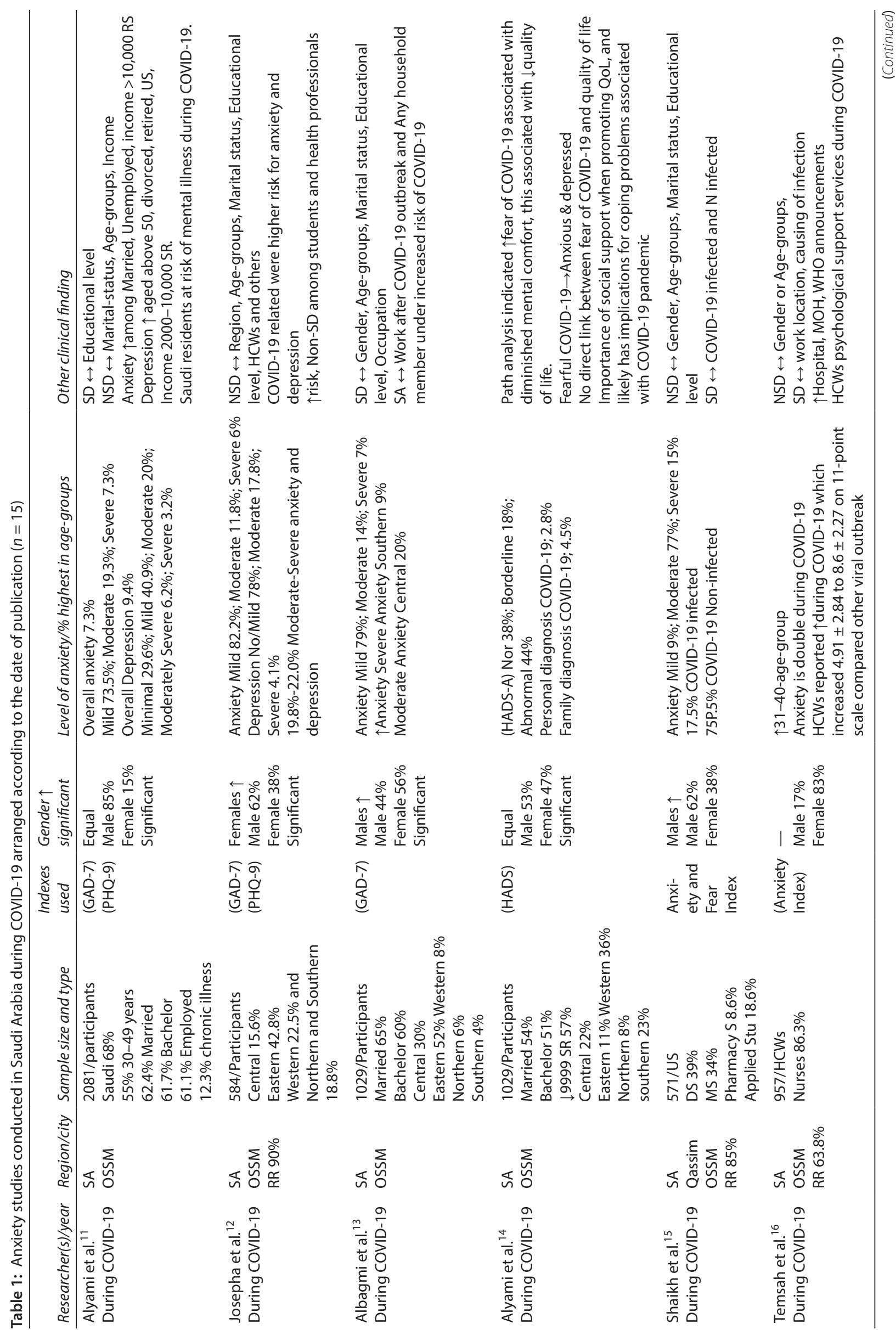




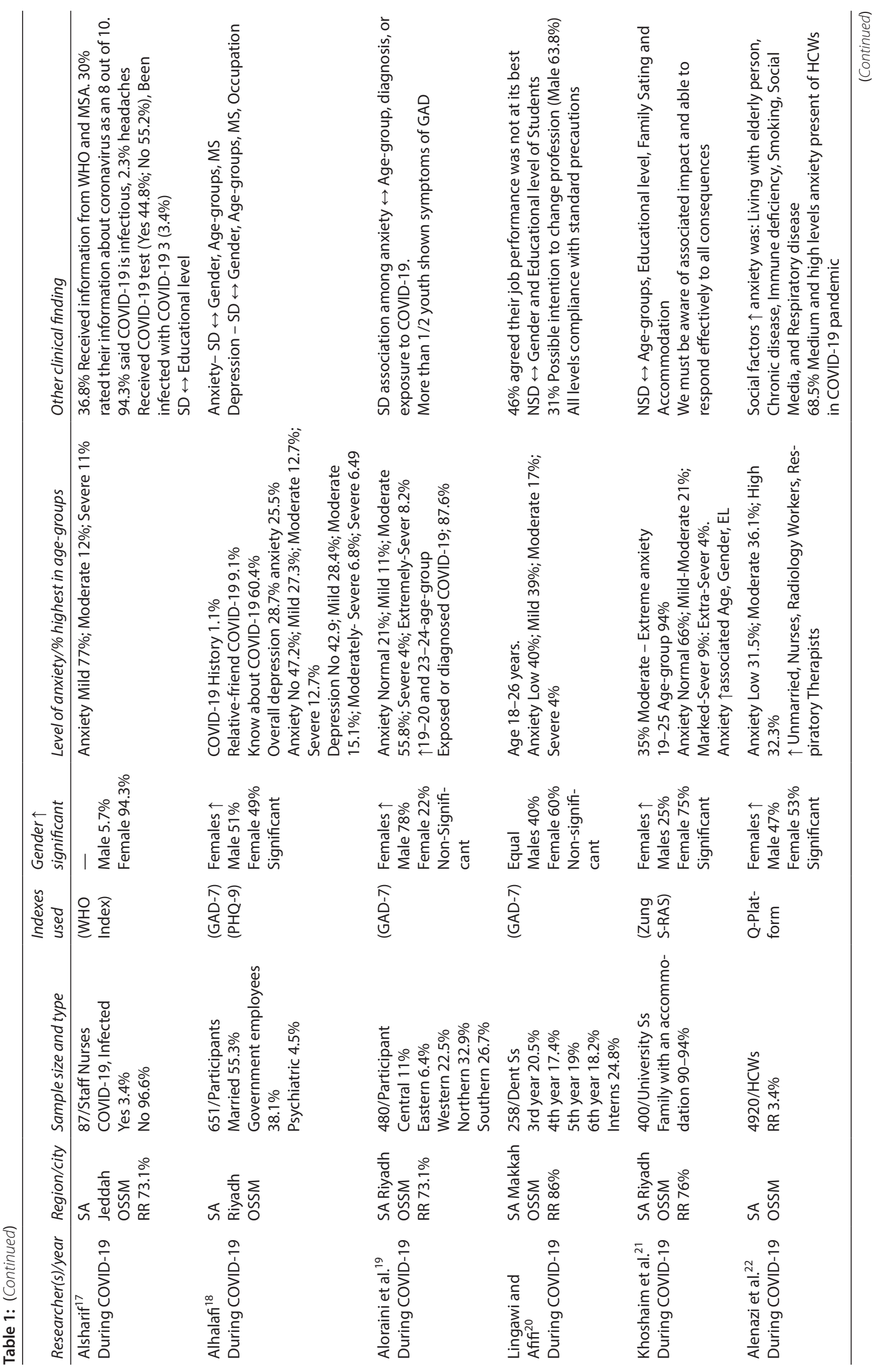




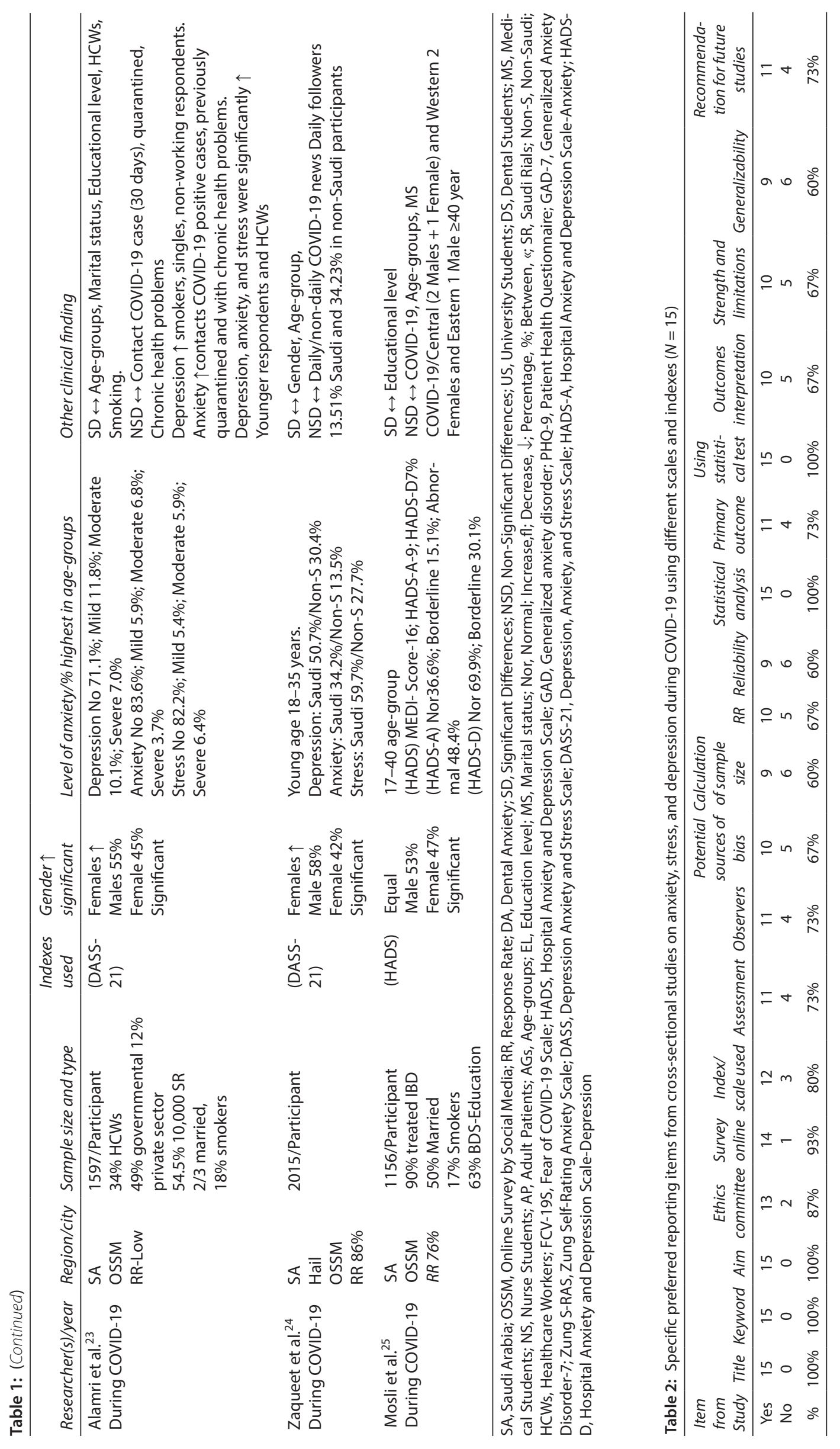


to males. This can be related to the women's higher depression and anxiety frequency percentages as well as biological gender variances, culture, diet, female hormonal fluctuations, or level of education. ${ }^{36}$ Other factors such as different assessment tools used and the timing of the study are important influencing factors as the psychological impact is expected to be much more intense at the beginning of the pandemic because little information was known about the disease. ${ }^{11}$ Furthermore, Wang et al. ${ }^{4}$ revealed that females were significantly associated with a higher psychological impact following the COVID-19 outbreak and had recorded levels of stress, anxiety, and depression. This finding is in complete agreement with studies mentioning that anxiety, depression, and stress are higher in female Saudi patients as compared to males. ${ }^{11,12,18,23-25}$ Also the results coincide with the study carried-out and published in Yemen by Alrubaiee et al. ${ }^{37}$

Health care workers (HCWs) had recorded a high level of anxiety with respect to COVID-19, with a wide range compared with the previous studies, such as local studies conducted by Temsah et al., Alsharif, and Alkhamees et al., 16,17,38 a study in Yemen by Alrubaiee et al., ${ }^{37}$ or international studies published by in different countries. ${ }^{39-43}$ Studies documented that anxiety levels were high among HCWs during the COVID-19 pandemic. ${ }^{16,38,43}$ The ratios were in the average percentages, counting the studies in this review. A recent literature review including 115 studies has concluded that the COVID-19 pandemic has a significant impact on the physical and mental health of HCWs. ${ }^{43}$ Some studies have shown that medical staff in their middle age were more worried about becoming infected with severe acute respiratory syndrome coronavirus 2 (SARS-CoV-2, the causative agent of COVID-19) and infecting their families compared with other age-groups. ${ }^{41,43}$

Two published studies mentioned ${ }^{15,17}$ that, a significant difference were recorded and observed between participant infected and non-infected with COVID-19.

Different from Wang et al., ${ }^{4}$ the study results ${ }^{19}$ show that educational level has no significant association with the psychological impact of COVID-19, a finding similar to a Chinese study by Cai, et al. ${ }^{44}$ One explanation is that most of the participants in the current study hold university degree or higher, and they all completed the online questionnaire by themselves, indicating they have access to online sources of information, thus attenuating the impact of educational background.

From Table 1, the respondents' percentage was slightly higher in males than females, which is comparable with the demographic profile of the previous studies directed at the beginning of 2020 preceding the COVID-19 outbreak in SA. ${ }^{40,42}$ Most respondents in this review were between the ages of 18 and 40 , and they showed higher level of anxiety. This is in parallel with the result obtained in South Asia during the COVID-19 pandemic. ${ }^{41,42}$ Thus, these findings may enable policymakers to announce numerous procedures and psychological involvements that can improve mental health throughout the existing pandemic. This table also shows that most of the conducted studies found non-significant differences between

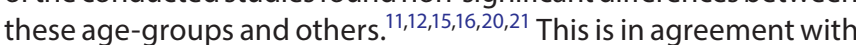
studies conducted in China published by Lai et al. and Kang et al. ${ }^{39,40}$ Elder groups are usually associated with depression rather than stress, which resulted in significant differences between elder age-groups and younger age-groups, as clearly shown in studies conducted in SA from Table $1^{13,23-25}$ Furthermore, the prevalence rate of anxiety was higher in the younger age-group, which agrees with our review as a higher score of depression was associated with individuals younger than 19 years.
The findings of this review stress the importance of adopting strategies/plans to mitigate the probable psychological consequences of COVID-19 pandemic among general and vulnerable residents. Many guidelines were delivered locally and worldwide, including in Saudi Arabia, to help alleviate some of these negative impacts, such as courses in schools or university, media shows, and campaigns.

This study has some limitations. First, the numbers gathered were from self-administered questionnaires conducted on an online device, so the participant number is somewhat low. The participants may offer information that meet the social expectation relatively than certainly, and the clinical significance may be unexpected. Second, this study does not exploit the psychological responses of COVID-19 patients or survivors as its primary focus is the general public.

\section{Conclusion}

From the several publications that assessed anxiety in different health centers, Universities, HCW hospitals, and specialized centers, anxiety represents a serious and extensive problem for HCWs and patients in the SA. In this systematic review, $45 \%$ of participants reported moderate to severe levels of anxiety. Groups vulnerable to anxiety were females, young adults, and University students. Lack of time for HCWs, cost of treatment outside governmental clinics, and unequal distribution of general health treatments might have played a role in aggravating anxiety among patients. Educational sessions and campaigns with training of HCWs and their teams, health education and social programs or seminars that encompass different populations in more cities throughout the kingdom are necessary to overcome this problem.

\section{ORCID ID}

Mohammed MAIMoaleem $\odot$ https://orcid.org/0000-0002-9623-261X

\section{References}

1. Ohman A. Fear and anxiety, evolutionary, cognitive, and clinical perceptions. In: Lewis M, Haviland JM (Ed.), Handbook of Emotions (3rd ed.). New York: The Gulliford Press; 2000: pp. 573-593.

2. Klingberg G, Broberg AG. Dental fear/anxiety and dental behavior management problems in children and adolescents: a review of prevalence and concomitant psychological factors. Int J Paediatr Dent 2007:17(6):391-406. DOI: 10.1111/j.1365-263X.2007.00872.x

3. Al-Naggar RA, Al-Naggar DH. Prevalence and associated factors of emotional disorder among Malaysian University students. Public Health 2012;4:11.

4. Wang C, Pan R, Wan X, et al. Immediate psychological responses, and associated factors during the initial stage of the 2019 coronavirus disease (COVID-19) epidemic among the general population in China. Int J Environ Res Public Health 2020;17:1729. DOI: 10.3390/ ijerph17051729

5. Huang JZ, Han MF, Luo TD, et al. Mental health survey of medical staff in a tertiary infectious disease hospital for COVID-19. Zhonghua Lao Dong Wei Sheng Zhi Ye Bing Za Zhi 2020; 38: 192-195. DOI: 10.3389/ fpsyt.2021.567381

6. World Health Organization. Coronavirus disease (COVID-19) situation report 132 2021. https://covid19.who.int/

7. World Stats. Coronavirus Worldwide Data 2021. https://www.worldstat.info/ (Accessed July 02.07.21).

8. Channel News Asia. Novel Coronavirus Map 2021. https://infographics. channel,news,asia.com/covid-19/map.html (Accessed 02.07.21).

9. 5271877 Total COVID-19 Cases and 8259 Death, MOH Says (Press Release), Ministry of Health: Riyadh, Saudi Arabia, 2021. 
10. Alyami MH, Naser AY, Orabi MAA, et al. Epidemiology of COVID-19 in the Kingdom of Saudi Arabia: an ecological study. Front Public Health. 2020;8:506. https://doi.org/10.3389/fpubh.2020.00506

11. Alyami HS, Naser AY, Dahmash EZ, et al. Depression and anxiety during the COVID-19 pandemic in Saudi Arabia: a cross-sectional study. Int J Clin Pract 2021;00:e14244. https://doi.org/10.1111/ ijcp.14244

12. Josepha R, Luccaa JM, Alshaybana D, et al. The immediate psychological response of the general population in Saudi Arabia during COVID-19 pandemic: a cross-sectional study. J Infect Public Health 2021;14:276-283. DOI: 10.1016/j.jiph.2020.11.017

13. Albagmi FM, AINujaidi HY, Al Shawan DS. Anxiety levels amid the COVID-19 lockdown in Saudi Arabia. Inter J General Med 2021;14:2161-2170. DOI: 10.2147/IJGM.S312465

14. Alyami M, de Albuquerque JV, Krägeloh CU, et al. Effects of fear of COVID-19 on mental well-being and quality of life among Saudi adults: a path analysis. Saudi J Med Med Sci 2021;9:24-30. DOI: 10.4103/sjmms.sjmms_630_20

15. Shaikh S, Mohsin SF, Agwan MAS, et al. COVID-19: fear and anxiety among healthcare students in Saudi Arabia: a cross-sectional study. Eur J Mol Clin Med 2021;8(3):1638-1647.

16. Temsah $\mathrm{MH}$, Al Huzaimi A, Alrabiaah A, et al. Changes in healthcare workers' knowledge, attitudes, practices, and stress during the COVID-19 pandemic. Medicine 2021;100(18):e25825. DOI: 10.1097/ MD. 0000000000025825

17. Alsharif $F$. Nurses' knowledge and anxiety levels toward COVID19 in Saudi Arabia. Nurs Rep 2021;11:356-363. DOI: 10.3390/ nursrep11020034

18. Alhalafi AH. Prevalence of anxiety and depression during the coronavirus disease 2019 pandemic in Riyadh, Saudi Arabia: a webbased cross-sectional survey. J Pharm Res Inter 2020;32(27):65-73.

19. Aloraini MI, Mohammad SM, Hejazi MS, et al. Prevalence of generalized anxiety disorder among Saudi youth during COVID19 pandemic in Saudi Arabia. Inter J Med Develop Count 2020;4(12):2322-2327. DOI: 10.24911/IJMDC.51-1605818315

20. LingawiHS, AfifiK.COVID-19associated stressamong dental students. Open Dent J 2020;14:555-562. DOI: 10.2174/1874210602014010554

21. Khoshaim HB, Al-Sukayt A, Chinna K, et al. Anxiety level of university students during COVID-19 in Saudi Arabia. Front Psychiatr 2020;11:579750. DOI: 10.3389/fpsyt.2020.579750

22. Alenazi TH, Bin Shimc NF, Alenazi MH, et al. Prevalence and predictors of anxiety among healthcare workers in Saudi Arabia during the COVID-19 pandemic. J Infect Public Health 2020;13:1645-1651. DOI: 10.1016/j.jiph.2020.09.001

23. Alamri HS, Algarni A, Shehata SF, et al. Prevalence of depression, anxiety, and stress among the general population in Saudi Arabia during COVID-19 pandemic. Int J Environ Res Public Health 2020;17:9183. DOI:10.3390/ijerph17249183

24. Zakou YM-A, Alreshidi FS, Elsaid RM, et al. The magnitude of COVID-19 related stress, anxiety and depression associated with intense mass media coverage in Saudi Arabia. AIMS Public Health 2020;7(3):664-678. DOI: 10.3934/publichealth.2020052

25. Mosli M, Alourfi M, Alamoudi A, et al. A cross-sectional survey on the psychological impact of the COVID-19 pandemic on inflammatory bowel disease patients in Saudi Arabia. Saudi J Gastroenterol 2020;26:263-271. DOI: 10.4103/sjg.SJG_220_20

26. AlDhelai TA, Al-Ahmari MM, Adawi HA, et al. Dental anxiety and fear among patients in Jazan, Kingdom of Saudi Arabia: a cross-sectional study. J Contemp Dent Pract 2021;22(5): 549-556. DOI: 10.5005/ jp-journals-10024-3066

27. Jumaymi AK, Faqehi WH, Hamdi SA, et al. Level of dental anxiety and its relation to khat chewing in Jazan population: a cross-sectional study. J Contemp Dent Pract 2020;21(3):253-260. DOI: 10.5005/ jp-journals-10024-2767
28. Al-Khalifa KS. Prevalence of dental anxiety in two major cities in the Kingdom of Saudi Arabia. Saudi J Med Med Sci 2015;3(2):135-140. DOI: 10.4103/1658-631X.156421

29. Al Jasser RA, Almashaan $G$, Alwaalan $H$, et al. Dental anxiety among dental, medical, and nursing students of two major universities in the central region of the Kingdom of Saudi Arabia: a cross-sectional study. BMC Oral Health 2019;19(1):56. DOI: 10.1186/s12903-019-0757-x

30. Moher D, Liberati, A, Tetzlaff J, et al. PRISMA Group. Preferred reporting items for systematic reviews and meta-analyses: the PRISMA statement. PLoS Med 2009;6:e1000097. DOI: 10.1371/journal. pmed.1000097

31. Herrero-Hernández S, López-Valverde N, Bravo M, et al. Root canal morphology of the permanent mandibular incisors by cone beam computed tomography: a systematic review. Appl Sci 2020;10:4914. DOI: 10.3390/app10144914

32. Zhou P, Yang XL, Wang XG, et al. A pneumonia outbreak associated with a new coronavirus of probable bat origin. Nature 2020;579:270-273. DOI: 10.1038/s41586-020-2012-7

33. Xiong J, Lipsitz O, Nasri F, et al. Impact of COVID-19 pandemic on mental health in the general population: a systematic review. J Affect Disord 2020;277:55-64. DOI: 10.1016/j.jad.2020.08.001

34. Al-Khathami A, Ogbeide D. Prevalence of mental illness among Saudi adult primary-care patients in Central Saudi Arabia. Saudi Med J 2002;23:721-724. DOI: 10.1038/s41598-018-21243-x

35. Lim GY, Tam WW, Lu Y, et al. Prevalence of depression in the community from 30 countries between 1994 and 2014. Sci Rep 2018;8:2861. DOI: 10.1038/s41598-018-21243-x

36. Albert PR. Why is depression more prevalent in women? J Psychiatry Neurosci 2015;40:219-221. DOI: 10.1016/S2215-0366(16)30263-2

37. Alrubaiee, GG, Al-Qalah TAH, Al-Aawar MSA. Knowledge, attitudes, anxiety, and preventive behaviours towards COVID-19 among health care providers in Yemen: an online cross-sectional survey. BMC Public Health 2020;20:1-11. DOI: 10.1186/s12889-020-09644-y

38. Alkhamees AA, Alrashed SA, Alzunaydi AA, et al. The psychological impact of COVID-19 pandemic on the general population of Saudi Arabia. Compr Psychiatr 2020;102:152192. DOI: 10.1016/j. comppsych.2020.152192

39. Lai J, Ma S, Wang Y, et al. Factors associated with mental health outcomes among health care workers exposed to coronavirus disease. JAMA Netw Open 2019;3:e203976. DOI: 10.1001/ jamanetworkopen.2020.3976

40. Kang L, Li Y, Hu S, et al. The mental health of medical workers in Wuhan, China dealing with the 2019 novel coronavirus. Lancet Psychiatr 2020;7:e14. DOI: 10.1016/S2215-0366(20)30047-X

41. Cai H, Tu B, Ma J, et al. Psychological impact and coping strategies of frontline medical staff in Hunan between January and March 2020 during the outbreak of coronavirus disease 2019 (COVID-19) in Hubei, China. Med Sci Monit 2020;26: e924171. DOI: 10.12659/MSM.924171

42. Mohindra R, Ravaki R, Suri $V$, et al. Issues relevant to mental health promotion in frontline health care providers managing quarantined/ isolated COVID19 patients. Asian J Psychiatr 2020;51:102084. DOI: 10.1016/j.ajp.2020.102084

43. de Pablo GS, Vaquerizo-Serrano J, Catalan A, et al. Impact of coronavirus syndromes on physical and mental health of health care workers: systematic review and meta-analysis. J Affect Disord 2020;275:48-57. DOI: 10.1016/j.ajp.2020.102084

44. Cai X, Hu X, Ekumi IO, et al. Psychological distress and its correlates among COVID-19 survivors during early convalescence across agegroups. Am J Geriatr Psychiatr 2020;28:1030-1039. DOI: 10.1016/j. jagp.2020.07.003 